\title{
ON A PROBLEM OF AXLER, CUCKOVIC AND RAO
}

\author{
GUANGFU CAO
}

(Communicated by Joseph A. Ball)

\begin{abstract}
In this note we show that if two Toeplitz operators on a Bergman space of the (Levi) pseudoconvex domain commute and the symbol of one of them is analytic and non-constant, then the other one is also analytic. This gives an affirmative answer of a problem of S. Axler, Z. Cuckovic and N. V. Rao (1999).
\end{abstract}

\section{INTRODUCTION}

Let $\Omega$ be a domain in $\mathbb{C}^{n}$ and $d V_{n}$ denote the Lebesgue measure on $\Omega$. The Bergman space $L_{a}^{2}(\Omega)$ is the subspace of $L^{2}\left(\Omega, d V_{n}\right)$ consisting of the squareintegrable functions that are analytic on $\Omega$. Write the orthogonal projection of $L^{2}\left(\Omega, d V_{n}\right)$ onto $L_{a}^{2}(\Omega)$ by $P$, for any bounded measurable function $\varphi$ on $\Omega$, define the Toeplitz operator $T_{\varphi}$ with symbol $\varphi$ on $L_{a}^{2}(\Omega)$ as

$$
T_{\varphi}(f)=P(\varphi f) \quad\left(\forall f \in L_{a}^{2}(\Omega)\right) .
$$

In the case of the Hardy space of the unit circle, Brown and Halmos [3] proved the following theorem.

Theorem 1. If the Toeplitz operators $T_{\varphi}$ and $T_{\psi}$ commute on the Hardy space, then (1) either both symbols are analytic, (2) both symbols are conjugate analytic, or (3) $a \varphi+b \psi$ is constant for some constants $a, b$ not both 0.

Cowen [4] and Thomson [8], [9] obtained more general results concerning which operators, not necessarily Toeplitz, commute with an analytic Hardy space Toeplitz operator on the Hardy space of the unit circle.

In [1], S. Axler, Z. Cuckovic and N. V. Rao proved the following theorem.

Theorem 2. Let $\Omega$ be a bounded open domain in the complex plane. Suppose that $\varphi$ is a non-constant bounded analytic function on $\Omega$ and that $\psi$ is a bounded measurable function on $\Omega$ such that $T_{\varphi}$ and $T_{\psi}$ commute. Then $\psi$ is analytic.

Their proof depends on the following approximation theorem due to Bishop [2].

Theorem 3. Let $\varphi$ be a non-constant bounded analytic function on $\Omega$. Then the norm-closed subalgebra of $L^{\infty}(\Omega, d A)$ generated by $\bar{\varphi}$ and the bounded analytic functions on $\Omega$ contain $C(\bar{\Omega})$.

\footnotetext{
Received by the editors August 17, 2006 and, in revised form, October 31, 2006.

2000 Mathematics Subject Classification. Primary 47B35.

Key words and phrases. Toeplitz operator, commutant of an operator.

Supported by National Natural Science Foundation of China.
} 
In the paper [1], the authors posed the following problem.

Problem. What is the situation on Bergman space in higher dimensions?

For the general domains, the problem has a negative answer. The main result [7] gives that on the bidisk $D^{2}, T_{z_{1}}$ commutes with $T_{\bar{z}_{2}}$ on the Bergman space. Hence the problem depends deeply on the geometric structure of domains. There are two great differences between one complex variable and several complex variables for handling the problem:

(1) If $\varphi$ is an analytic function on the unit disk $D$, then for any $\lambda \in \mathbb{C}$, $\{z \mid \varphi(z)=\lambda\}$ has no limit point in $D$. However, if $\varphi$ is an analytic function on the unit ball $B_{n}$, then for any $\lambda \in \mathbb{C},\{z \mid \varphi(z)=\lambda\}$ may be a $k$-dimensional manifold $(k<n)$.

(2) In the case of a domain for several complex variables, if $f$ is a $(p, q)$-form with $C^{\infty}$-coefficients on the domain which satisfies $\bar{\partial} f=0$, there may be no $(p, q-1)$-form $g$ such that $\bar{\partial} g=f$. In general, one needs the domain to be at least pseudoconvex. Here we say that $\Omega$ is (Levi) pseudoconvex if $\Omega \Subset \mathbb{C}^{n}$ has $C^{2}$-boundary (where $\Omega \Subset \mathbb{C}^{n}$ means that $\Omega$ is relatively compact in $\mathbb{C}^{n}$; that is, the closure $\bar{\Omega}$ of $\Omega$ is a compact subset of $\mathbb{C}^{n}$ ) i.e., there is a defining function $\rho$ on a neighborhood $W$ of $\partial \Omega$ so that $\rho$ is of class $C^{2}, \Omega \cap W=\{z \in W \mid \rho(z)<0\}$, and $\operatorname{grad} \rho \neq 0$ on $\partial \Omega$, where grad $\rho$ denotes the gradient of $\rho$, and for each $z \in \partial \Omega$ and each $w \in \mathbb{C}^{n}$ satisfying $\sum_{j=1}^{n}\left[\frac{\partial \rho(z)}{\partial z_{j}}\right] w_{j}=0$, one has

$$
\sum_{j, k=1}^{n} \frac{\partial^{2} \rho(z)}{\partial z_{j} \partial \overline{z_{k}}} w_{j} \overline{w_{k}} \geq 0
$$

for any defining function $\rho$ for $\Omega$. For more details about pseudoconvex domains, see [5].

The key to solving the problem is to establish an analogue of Bishop's theorem in higher dimension. In the present note, we obtain a weak form of Bishop's theorem in the case of $\mathbb{C}^{n}$, and hence give an affirmative answer to the problem of Axler, Cuckovic and Rao.

\section{MAin RESUlt}

In this section we will state and prove the main result in the paper. First we need the following lemma, which is Corollary 4.6.12 in [5].

Lemma 4. If $\Omega \subseteq \mathbb{C}^{n}$ is pseudoconvex and $f$ is a $(p, q+1)$ form on $\Omega$ with $C^{\infty}$-coefficients and satisfying $\bar{\partial} f=0$, then there is $a(p, q)$ form $u$ on $\Omega$ with $C^{\infty}$-coefficients satisfying $\bar{\partial} u=f$.

The $(p, q)$ form $f$ satisfying $\bar{\partial} f=0$ is also called a close $(p, q)$ form. The following theorem extends Bishop's theorem [2] to pseudoconvex domains in $\mathbb{C}^{n}$ in a weak form. But it is good enough for us to solve the problem of Axler, Cuckovic and Rao.

For any bounded pseudoconvex domain $\Omega$ in $\mathbb{C}^{n}$, let $H^{\infty}(\Omega)[\bar{\varphi}]$ denote the subalgebra of $L^{\infty}\left(\Omega, d V_{n}\right)$ generated by $\bar{\varphi}$ and $H^{\infty}(\Omega)$.

Theorem 5. Suppose $\Omega$ is a bounded pseudoconvex domain in $\mathbb{C}^{n}$ and $\varphi \in H^{\infty}(\Omega)$ is non-constant on $\Omega$. Then $C(\bar{\Omega}) \subset L_{a}^{2}(\Omega)[\bar{\varphi}]$, where $L_{a}^{2}(\Omega)[\bar{\varphi}]$ denotes the closure in $L^{2}\left(\Omega, d V_{n}\right)$ of the algebra $H^{\infty}(\Omega)[\bar{\varphi}]$. 
Proof. Take $g$ in $C(\bar{\Omega})$ and assume that $\varphi$ is non-constant on $\Omega$. We will show that $g$ is contained in $L_{a}^{2}(\Omega)[\bar{\varphi}]$.

Without loss of generality, assume $\|\varphi\|_{\infty} \leq 1$. For any $\lambda \in D$, write

$$
E_{\lambda}=\{z \in \Omega \mid \varphi(z)=\lambda\}
$$

then $V_{n}\left(E_{\lambda}\right)=0$. For arbitrary $\varepsilon>0$, let $U_{1}, U_{2}$ be open sets which satisfy $E_{\lambda} \cup$ $\partial \Omega \subset U_{1}, \overline{U_{1}} \subset U_{2}$ and $V_{n}\left(U_{2}-E_{\lambda} \cup \partial \Omega\right)=V_{n}\left(U_{2}\right)<\varepsilon$. By Corollary 1.2.6 in [6], we see easily that there exists $g_{\lambda \varepsilon} \in C^{\infty}(\bar{\Omega})$ such that

$$
\left.g_{\lambda \varepsilon}\right|_{\Omega-\overline{U_{2}}}=\left.g\right|_{\Omega-\overline{U_{2}}},\left.\quad g_{\lambda \varepsilon}\right|_{\overline{U_{1}}}=0, \quad\left\|g_{\lambda \varepsilon}\right\|_{\infty} \leq\|g\|_{\infty} .
$$

Obviously, $\frac{\bar{\partial} g_{\lambda \varepsilon}}{\varphi-\lambda}$ is a $(0,1)$-form with $C^{\infty}$-coefficients and $\operatorname{supp}\left(\frac{\bar{\partial} g_{\lambda \varepsilon}}{\varphi-\lambda}\right) \subset \Omega$. Let $\Omega_{\delta}$ be a pseudoconvex domain containing $\bar{\Omega}$ such that $g_{\lambda \varepsilon}$ is well defined on $\Omega_{\delta}$ (such a $\delta$ exists because $g_{\lambda \varepsilon} \in C^{\infty}(\bar{\Omega})$. In fact, if $\rho$ is the defining function for $\Omega$, then we may choose $\delta$ sufficiently small so that $\rho_{\delta}=\rho-\delta$ is the defining function for $\left.\Omega_{\delta}\right)$. Note $\frac{\bar{\partial} g_{\lambda \varepsilon}}{\varphi-\lambda}$ is a $\bar{\partial}$ close $(0,1)$-form; hence there exists a $C^{\infty}$-function $h_{\lambda \varepsilon}$ on $\Omega_{\delta}$ which satisfies

$$
\bar{\partial} h_{\lambda \varepsilon}=\frac{\bar{\partial} g_{\lambda \varepsilon}}{\varphi-\lambda}
$$

by Lemma 4 . Therefore $g_{\lambda \varepsilon}-(\varphi-\lambda) h_{\lambda \varepsilon}$ is a holomorphic function on $\Omega$, and $\bar{\partial} h_{\lambda \varepsilon}$ also has support contained in $\Omega$. This shows that $h_{\lambda \varepsilon}$ is analytic in a neighborhood of $\partial \Omega$.

Now assume $\left\{\lambda_{i}\right\}_{i=1}^{m}$ is a finite collection of points in $\bar{D}, \widetilde{\varepsilon_{i}}<\varepsilon, \Delta_{i}$ is the open square with center $\lambda_{i}$, diameter $\frac{\widetilde{\varepsilon_{i}}}{\left\|h_{\lambda_{i} \varepsilon}\right\|_{\infty}}$ which satisfies $\bigcup_{i=1}^{m} \Delta_{i} \supset \bar{D}$. Set $\varepsilon_{i}=\frac{\widetilde{\varepsilon_{i}}}{\left\|h_{\lambda_{i} \varepsilon}\right\|_{\infty}}$, and take a partition of unity $\left\{P_{j}(x, y)\right\}_{j=1}^{m}(z=x+i y)$ on $\bar{D}$ such that $\operatorname{supp} P_{j} \subset \Delta_{j}$. Since $\varphi \in H^{\infty}(\Omega)$, clearly, $\operatorname{Re} \varphi=\frac{\varphi+\bar{\varphi}}{2}, \operatorname{Im} \varphi=\frac{\varphi-\bar{\varphi}}{2 i}$ are in $H^{\infty}[\bar{\varphi}]$. Define

$$
f(z)=\sum_{j=1}^{m}\left[g_{\lambda_{j} \varepsilon}(z)-h_{\lambda_{j} \varepsilon}(z)\left(\varphi(z)-\lambda_{j}\right)\right] P_{j}(\operatorname{Re} \varphi(z), \operatorname{Im} \varphi(z)) .
$$

We see that $f \in H^{\infty}(\Omega)[\bar{\varphi}]$ and

$$
\begin{aligned}
& |g(z)-f(z)| \leq \sum_{j=1}^{m}\left|g(z)-g_{\lambda_{j} \varepsilon}(z)\right| \cdot\left|P_{j}(\operatorname{Re} \varphi(z), \operatorname{Im} \varphi(z))\right| \\
& \quad+\sum_{j=1}^{m}\left|h_{\lambda_{j} \varepsilon}(z)\right| \cdot\left|\varphi(z)-\lambda_{j}\right| \cdot\left|P_{j}(\operatorname{Re} \varphi(z), \operatorname{Im} \varphi(z))\right| .
\end{aligned}
$$

Since

$$
\begin{gathered}
\sum_{j=1}^{m}\left|h_{\lambda_{j} \varepsilon}(z)\right| \cdot\left|\varphi(z)-\lambda_{j}\right| \cdot\left|P_{j}(\operatorname{Re\varphi }(z), \operatorname{Im} \varphi(z))\right| \\
=\sum_{j=1}^{m}\left|h_{\lambda_{j} \varepsilon}(z)\right| \cdot\left|\varphi(z)-\lambda_{j}\right| \cdot\left|P_{j}(\operatorname{Re} \varphi(z), \operatorname{Im} \varphi(z))\right| \cdot \chi_{\left\{\left|\varphi(z)-\lambda_{j}\right|<\varepsilon_{j}\right\}} \\
+\sum_{j=1}^{m}\left|h_{\lambda_{j} \varepsilon}(z)\right| \cdot\left|\varphi(z)-\lambda_{j}\right| \cdot\left|P_{j}(\operatorname{Re} \varphi(z), \operatorname{Im} \varphi(z))\right| \cdot \chi_{\left\{\left|\varphi(z)-\lambda_{j}\right| \geq \varepsilon_{j}\right\}}
\end{gathered}
$$


(where $\chi_{E}$ denotes the characteristic function of the set $E$ ), and

$$
P_{j}(x, y)=0 \quad \text { for any } \quad(x, y) \notin \Delta_{j},
$$

we have that

$$
\begin{gathered}
\sum_{j=1}^{m}\left|h_{\lambda_{j} \varepsilon}(z)\right| \cdot\left|\varphi(z)-\lambda_{j}\right| \cdot\left|P_{j}(\operatorname{Re} \varphi(z), \operatorname{Im} \varphi(z))\right| \\
=\sum_{j=1}^{m}\left|h_{\lambda_{j} \varepsilon}(z)\right| \cdot\left|\varphi(z)-\lambda_{j}\right| \cdot\left|P_{j}(\operatorname{Re} \varphi(z), \operatorname{Im} \varphi(z))\right| \cdot \chi_{\left\{\left|\varphi(z)-\lambda_{j}\right|<\varepsilon_{j}\right\}} \\
\leq \sum_{j=1}^{m} \varepsilon\left|P_{j}(\operatorname{Re} \varphi(z), \operatorname{Im} \varphi(z))\right| \leq \varepsilon .
\end{gathered}
$$

On the other hand,

$$
\begin{aligned}
& \int_{\Omega}\left[\sum_{j=1}^{m}\left|g(z)-g_{\lambda_{j} \varepsilon}(z)\right| \cdot\left|P_{j}(\operatorname{Re} \varphi(z), \operatorname{Im} \varphi(z))\right|\right]^{2} d V_{n} \\
= & \int_{\Omega \cap U_{2}}\left[\sum_{j=1}^{m}\left|g(z)-g_{\lambda_{j} \varepsilon}(z)\right| \cdot\left|P_{j}(\operatorname{Re} \varphi(z), \operatorname{Im} \varphi(z))\right|\right]^{2} d V_{n} .
\end{aligned}
$$

Note that

$$
\begin{gathered}
\sum_{j=1}^{m}\left|g(z)-g_{\lambda_{j} \varepsilon}(z)\right| \cdot\left|P_{j}(\operatorname{Re} \varphi(z), \operatorname{Im} \varphi(z))\right| \\
\leq\left\|g-g_{\lambda_{j} \varepsilon}\right\|_{\infty} \sum_{j=1}^{m}\left|P_{j}(\operatorname{Re} \varphi(z), \operatorname{Im} \varphi(z))\right| \\
=\left\|g-g_{\lambda_{j} \varepsilon}\right\|_{\infty} \leq 2\|g\|_{\infty} .
\end{gathered}
$$

Hence

$$
\begin{gathered}
\int_{\Omega \cap U_{2}}\left[\sum_{j=1}^{m}\left|g(z)-g_{\lambda_{j} \varepsilon}(z)\right| \cdot\left|P_{j}(\operatorname{Re} \varphi(z), \operatorname{Im} \varphi(z))\right|\right]^{2} d V_{n} \\
\leq 4\|g\|_{\infty}^{2} V_{n}\left(U_{2} \cap \Omega\right)<4\|g\|_{\infty}^{2} \varepsilon, \\
\int_{\Omega \cap U_{2}}\left[\sum_{j=1}^{m}\left|g(z)-g_{\lambda_{j} \varepsilon}(z)\right| \cdot\left|P_{j}(\operatorname{Re} \varphi(z), \operatorname{Im} \varphi(z))\right|\right] d V_{n} \\
\leq 2\|g\|_{\infty} V_{n}\left(U_{2} \cap \Omega\right)<2\|g\|_{\infty} \varepsilon .
\end{gathered}
$$

Further

$$
\begin{aligned}
\int_{\Omega}|g(z)-f(z)|^{2} d V_{n} & \leq 4\|g\|_{\infty}^{2} V_{n}\left(U_{2} \cap \Omega\right)+4 \varepsilon\|g\|_{\infty} V_{n}\left(U_{2} \cap \Omega\right)+\varepsilon^{2} \\
& \leq \varepsilon\left[4\|g\|_{\infty}^{2}+4 \varepsilon\|g\|_{\infty}+\varepsilon\right] .
\end{aligned}
$$

This gives that $g \in L_{a}^{2}(\Omega)[\bar{\varphi}]$, to complete the proof.

We are ready to prove our main result.

Theorem 6. If $\varphi$ is a non-constant bounded analytic function on $\Omega$, and $\psi$ is a bounded measurable function on $\Omega$ such that $T_{\varphi}$ and $T_{\psi}$ commute, then $\psi$ is analytic. 
Proof. Our proof is similar to that in [1]. Suppose $\varphi$ is a non-constant bounded analytic function on $\Omega$, and $\psi$ is a bounded measurable function on $\Omega$ such that $T_{\varphi} T_{\psi}=T_{\psi} T_{\varphi}$. Write $\psi=f+u$ with $f \in L_{a}^{2}(\Omega)$, and $u \in L^{2}\left(\Omega, d V_{n}\right) \ominus L_{a}^{2}(\Omega)$. For a non-negative integer $n$, an easy calculation gives

$$
T_{\varphi^{n}} T_{\psi} 1=\varphi^{n} P(f+u)=\varphi^{n} f
$$

and

$$
T_{\psi} T_{\varphi^{n}} 1=P\left(f \varphi^{n}+u \varphi^{n}\right)=f \varphi^{n}+P\left(u \varphi^{n}\right) .
$$

Since $T_{\varphi} T_{\psi}=T_{\psi} T_{\varphi}$, by induction, we obtain easily that

$$
T_{\varphi^{n}} T_{\psi}=T_{\psi} T_{\varphi^{n}} .
$$

Thus $P\left(u \varphi^{n}\right)=0$. Consequently, if $h \in L_{a}^{2}(\Omega)$, we have

$$
0=\left\langle h, u \varphi^{n}\right\rangle=\int_{\Omega} \bar{u} h \overline{\varphi^{n}} d V_{n} .
$$

Theorem 5 gives that

$$
\int_{\Omega} \bar{u} g d V_{n}=0
$$

for every $g \in C(\bar{\Omega})$.

On the other hand, $C(\bar{\Omega})$ is dense in $L^{2}(\Omega)$. Hence $u=0$. Thus we have that $\psi=f$, and hence $\psi$ is analytic, to complete the proof.

Theorem 6 immediately gives the following corollary.

Corollary 7. Suppose $\Omega$ is a pseudoconvex domain, $\varphi, \psi \in H^{\infty}$. Then $T_{\varphi} T_{\psi}^{*}=$ $T_{\psi}^{*} T_{\varphi}$ if and only if either $\varphi$ or $\psi$ is constant.

\section{REFERENCES}

[1] Sheldon Axler, Zeljko Cuckovic and N. V. Rao, Commutants of analytic Teoplitz operators on the Bergman space, Proc. Amer. Math. Soc., 128 (1999) 1951-1953. MR1694299 (2000m:47035)

[2] Christopher J. Bishop, Approximating continuous functions by holomorphic and harmonic functions, Trans. Amer. Math. Soc., 311 (1989) 781-811. MR961619 (89j:30051)

[3] A. Brown and P. R. Halmos, Algebraic properties of Toeplitz operators, J. Reine Angew. Math., 213 (1964) 89-102. MR0160136 (28:3350)

[4] Carl C. Cowen, The commutant of an analytic Toeplitz operator, Trans. Amer. Math. Soc., 239 (1978) 1-31. MR0482347 (58:2420)

[5] Steven G. Krantz, Function theory of several complex variables, New York, Chichester, Brisbane, Toronto, Singapore 1982. MR635928 (84c:32001)

[6] R. Narasimhan, Analysis on real and complex manifolds, North-Holland, Inc. 1973. MR832683 (87c:58002)

[7] S. Sun and D. Zheng, Toeplitz operators on the polydisk, Proc. Amer. Math. Soc., 124 (1996) 3351-3356. MR1328380 (97a:47038)

[8] James E. Thomson, The commutant of a class of analytic Toeplitz operators, Amer. J. Math., 99 (1977) 522-529 MR0461196 (57:1181)

[9] James E. Thomson, The commutant of a class of analytic Toeplitz operators II, Indiana Univ. Math. J., 25 (1976) 793-800. MR0417843 (54:5891)

Department of Mathematics, Guangzhou University, Guangzhou 510006, People's RePUBLIC OF CHINA

E-mail address: guangfucao@163.com 